\title{
A vigilância em saúde e o planejamento nas equipes de atenção primária em saúde: revisão narrativa
}

\author{
Health surveillance and planning in primary health care teams: narrative review
}

Vigilancia y planificación de la salud en los equipos de atención primaria de salud: revisión narrativa

Ilka Lorena de Oliveira Farias Costa ${ }^{1 *}$; Creusa Barbosa dos Santos Trindade"; Elem Cristina Rodrigues Chaves'; Ilma Pastana Ferreira1', Sérgio Beltrão de Andrade Lima²; Fátima Bastos da Costa $^{1}$; Maria Helena Rodrigues de Mendonça ${ }^{3}$; Rafael Lourenço da Silva Neto ${ }^{1}$.

\section{RESUMO}

Objetivo: Analisar através de uma revisão narrativa sobre o panorama atual do processo integração da Atenção Primária em Saúde e a Vigilância em Saúde e sua contribuição no processo de planejamento e avaliação em saúde. Revisão bibliográfica: O Sistema Único de Saúde, a partir da Lei Orgânica de Saúde (8.080/90), surge com princípios garantindo a universalização e descentralização da saúde sendo reconhecido por seus benefícios, todavia, torna-se um desafio para gestão devido sua fragilidade e subfinanciamento, influenciando o processo de planejamento e avaliação na atenção primária. O SUS possibilitou criar a Secretaria de Vigilância em Saúde, sendo um marco na descentralização de ações e organização no processo de avaliar e planejar na atenção básica, entretanto, embora existam estratégias para integrar a Vigilância à atenção primária, o mesmo não foi realizado de forma eficaz, caracterizando um obstáculo na identificação de determinantes saúde-doença, contrapondo-se aos princípios propostos na Lei № 8.080. Considerações finais: Frente aos desafios da integração da Vigilância em Saúde à Saúde básica e a gestão, são necessárias inovações nos métodos de planejar e avaliar para auxiliar decisão de determinada problemática e auxiliar na reorganização do trabalho, ações e serviços de saúde.

Palavras-chave: Vigilância em saúde, Atenção primária, Gestão em saúde.

\section{ABSTRACT}

Objective: To analyze through a narrative review about the current panorama of the integration process of Primary Health Care and Health Surveillance and its contribution in the health planning and evaluation process. Bibliographic review: The Unified Health System, based on the Organic Health Law $(8,080 / 90)$, emerges with principles ensuring the universalization and decentralization of health being recognized for its benefits, however, it becomes a challenge for management due to its fragility and underfunding, influencing the planning and evaluation process in primary care. The SUS made it possible to create the Health Surveillance Secretariat, being a milestone in the decentralization of actions and organization in the process of evaluating and planning in primary care, however, although there are strategies to integrate surveillance into primary care, it was not effectively performed, characterizing an obstacle in the identification of healthdisease determinants, in contrast to the principles proposed in Law No. 8,080. Final considerations: Final Faced with the challenges of integrating Health Surveillance into basic health and management, innovations are needed in the methods of planning and evaluating to assist decision-making of a given problem and assist in the reorganization of work, actions and health services.

Key words: Health surveillance, Primary care, Health management.

1Santa Casa de Misericórdia do Pará, Belém - PA. *E-mail: ilka_lorena@yahoo.com.br

${ }^{2}$ Centro Universitário Metropolitano da Amazônia, Belém - PA.

${ }^{3}$ Centro Universitário FIBRA, Belém - PA. 


\section{RESUMEN}

Objetivo: Analizar a través de una revisión narrativa sobre el panorama actual del proceso de integración de la Atención Primaria de Salud y Vigilancia de la Salud y su contribución en el proceso de planificación y evaluación de la salud. Revisión bibliográfica: El Sistema Unificado de Salud, basado en la Ley Orgánica de Salud (8.080/90), surge con principios que garantizan la universalización y descentralización de la salud que se reconoce por sus beneficios, sin embargo, se convierte en un desafío para la gestión debido a su fragilidad y infrafinanciación, influyendo en el proceso de planificación y evaluación en la atención primaria. EI SUS ha hecho posible la creación de la Secretaría de Vigilancia sanitaria, siendo un hito en la descentralización de las acciones y la organización en el proceso de evaluación y planificación en la atención primaria, sin embargo, aunque existen estrategias para integrar la vigilancia en la atención primaria, no se realizó efectivamente, caracterizando un obstáculo en la identificación de los determinantes de la salud-enfermedad, en contraste con los principios propuestos en la Ley No 8.080. Consideraciones finales: Ante los desafíos de la integración de la vigilancia sanitaria en la salud y la gestión básicas, se necesitan innovaciones en los métodos de planificación y evaluación para ayudar a la toma de decisiones de un problema determinado y ayudar en la reorganización del trabajo, las acciones y los servicios de salud.

Palabras clave: Vigilancia de la salud, Atención primaria, Gestión de la salud.

\section{INTRODUÇÃO}

O Ministério da Saúde, através da portaria № 1.378 de 9 de julho de 2013, conceitua a Vigilância em Saúde (VS) como uma atuação sistemática da coleta, análise e disseminação de dados referentes a saúde, realizada de forma contínua, surge com intuito de planear e implementar ações de políticas públicas para promoção, prevenção e recuperação da saúde da população (BRASIL, 2013). Tal definição remete as transformações do modelo de atenção à saúde existente na década de 1970 através da Reforma Sanitária Brasileira (RSB), os quais direcionaram importantes mudanças no setor de vigilância.

Segundo Pinafo E, et al. (2016), a descentralização da gestão em saúde, implantada em 1988 (Lei no 8.080), ao permitir a ampliação e transferências de recursos aos estados e municípios, não visou a distribuição das funções e competências da gestão do sistema entre entes federados, tal autonomia políticoadministrativa representou um grande desafio para os municípios no sentido a capacidade de desenvolver estas ações. De acordo com IPEA (2018), os processos de planejamento e avaliação devem fazer parte da gestão de saúde em todos os seus níveis, da unidade básica de saúde aos serviços mais complexos, fazendo parte das estratégias com metas a serem perseguidas tanto no nível tático como operacional (IPEA, 2018).

No Brasil, a evolução da Atenção Primária a Saúde (APS) é visível no sentido da ampliação da atuação de equipes de saúde, entretanto, o processo de promoção a saúde permanece complexo e desafiador devido ao déficit no financiamento, a inadequada gestão física das unidades básicas e as dificuldades de planejamento para integrar a atenção primária com vista na continuidade e gerenciamento de recursos (BOUSQUAT AEM, et al., 2017; FACCHINI LA, et al., 2018). Referente as unidades básicas de saúde (UBS), estas estão inseridas na APS e são entendidas como estratégia de organização do sistema de atenção à saúde mais compatível com a proposta das Redes de Atenção à Saúde (FACCHINI LA, et al., 2018).

No âmbito da APS, a Estratégia de Saúde da Família (ESF) foi adotada no Brasil como modelo de atenção a fim de alcançar o conceito de APS definida na Conferência de Alma Ata em 1978. Desde então a ESF passa, ao longo do tempo, por uma amplitude em seu foco deixando de ser apenas a porta de entrada do Sistema Único de Saúde, mas transformando-se em uma verdadeira estratégia de reorganização do SUS, oferecendo melhoria dos indicadores de saúde, promovendo maior assistência à população e qualificar a saúde básica (FACCHINI LA, 2018; VALADÃO APS, et al., 2019). As Unidades de Saúde da Família (USF) passam agora a reafirmar seu papel, coordenando o cuidado e conhecendo seu território e as singularidades a fim de aperfeiçoar seus processos de trabalho com protagonismo das equipes de saúde e da população que lhe são confiadas (BRASIL, 2010; VALADÃO APS, et al., 2019). 
A Política Nacional da Atenção Básica, através da Portaria no 2.436 de 21 de setembro de 2017, no Art. 4º , configura a Saúde da Família como plano prioritário para consolidação da Atenção Básica e, a respeito da integração entre Vigilância em Saúde e Atenção Básica, o Art. $5^{\circ}$ caracteriza essencial para o alcance de resultados satisfatórios visando às necessidades de saúde da população e a integralidade da atenção à saúde, além disso, visa estabelecer processos que considerem os determinantes, os riscos e danos à saúde, na perspectiva da intra e intersetorialidade (BRASIL, 2017).

Nesta perspectiva, a integração entre a Vigilância em Saúde e Atenção Básica deve propiciar o uso de ferramentas da vigilância no desenvolvimento de habilidades de programação e planejamento, organizando processo de trabalho das equipes, melhorando o acesso e o resultado de indicadores de saúde (BRASIL, 2010; SOUZA MMO e GUEDES JPF, 2013).

Ressalta que a integração da Vigilância em Saúde com a APS vem sendo amplamente debatida e, a recente Resolução no 588, de 12 de julho de 2018 que considera as deliberações da 1a Conferência Nacional de Vigilância em Saúde, trazà tona debates acerca da importância de se implementar ações deste setor no cotidiano das equipes de atenção primária à saúde a fim de e garantir a integralidade do cuidado (BRASIL, 2018). No Entanto, tal fato é considerado um desafio para a gestão do SUS pela não integração da Vigilância em Saúde a APS, pois ainda se observa uma Vigilância em Saúde centralizada.

Este artigo traz a evolução na atuação da Vigilância em Saúde e a importância de implementar a integração deste setor com a Atenção Primária em Saúde no sentido de melhorar os processos de planejamento e avaliação das equipes de saúde. Trata-se de processos relativamente novos dentro das gestões municipais que tiveram que assumir tais tarefas gerenciais a partir da municipalização. Nesse contexto, a vigilância em saúde traz importantes contribuições no sentido de possibilitar o diagnóstico situacional de determinado território, sendo ponto de partida na implementação de ações e serviços de saúde que sejam condizentes com as reais necessidades de uma população em um território.

O objetivo do presente artigo foi analisar através de uma revisão narrativa sobre o panorama atual do processo de integração da Atenção Primária em Saúde e a Vigilância em Saúde e sua contribuição no processo de planejamento e avaliação em saúde.

\section{REVISÃO BIBLIOGRÁFICA}

\section{O desafio da descentralização e o processo de planejar e avaliar}

O SUS nasce com um conjunto de princípios e diretrizes abrangendo uma visão ampla da saúde como direito do cidadão e dever do Estado, tal garantia é estabelecida na Lei Orgânica da Saúde 8.080/ 90 com a universalização do direito à saúde, redistribuição de responsabilidades entre os vários níveis de governo levando a descentralização e a integralidade da atenção à saúde com usuário participativo garantindo o controle social, e que foi então complementada com a Lei Orgânica da Saúde 8.142/90 dando orientações quanto à operacionalização desse sistema, inclusive definindo regras na realização de conferências de saúde e regulamentando os conselhos municipais e definindo regras para o financiamento da União (BRASIL, 1990; BRASIL, 2017).

Giovanella $L$ (2018) reconhece os benefícios trazidos com a descentralização da atenção à saúde, destacando a acessibilidade, redução da disparidade e melhoria no estado saúde da população, entretanto, expõeos atuais desafios e ameaças no quesito gestão da saúde proporcionado pelo governo ilegítimo diante aos direitos sociais.

Lorenzetti J, et al. (2014), em seu estudo, colocam "as fragilidades de gestão e sub-financiamento têm emergido como problemas relevantes em análises de estudiosos e gestores". E essa fragilidade gerencial atinge vários níveis de atenção em saúde de modo que os processos de planejamento e avaliação ainda são frágeis e diversas vezes não utilizados na prática de gestores e trabalhadores do SUS como ferramenta que propicie a aproximação de informações que norteiam políticas de saúde no âmbito municipal. Polati AM, et al. (2016) corrobora tal fragilidade ao relatar o sub-financiamento e o repasse defasado aos municípios com altas perdas de recursos ao sistema de saúde. Diante a autonomia no campo da saúde, remete a 
essencial gestão de saúde se empoderar dos processos de gestão a fim de administrar e operacionalizar o SUS de modo a refletir suas dificuldades e necessidades e assim, traçar os caminhos a percorrer.

O princípio da gestão ainda está fundamentado em estratégias tradicionais; alicerçada no individualismo e, ainda, de forma não ativa entre setores da saúde. Além disso, dados disponíveis através dos sistemas de informação, que podem subsidiar a tomada de decisão dos gestores, não são utilizados de modo que favoreça tal movimento, ficando limitado a meros registros. Confronta-se a essa realidade, a dificuldade real de profissionais e gestores de saúde de apresentar e tornar o processo de planejamento e avaliação uma prática que subsidie a tomada de decisão (PINHEIRO ALS, et al., 2016; PINAFO E, et al., 2016; GIOVANELLA L, 2018).

Carvalho ALB e Shimizu HE (2017) alegam fatores referentes ao processo de avaliação em saúde como as mudanças nos procedimentos administrativos do SUS. E ainda dispõe que, para o Ministério da Saúde, "a avaliação em saúde apresenta-se, ainda, por intermédio de processos incipientes, pouco incorporados às práticas, possuindo, quase sempre, um caráter mais prescritivo e burocrático". Segundo Furtado JP, et al. (2018), a avaliação em saúde, quando realizada de forma correta, constitui-se um mecanismo de contribuição para renovação dos serviços, favorecendo a autonomia e a capacidade de analisar e intervir nos setores.

Miranda AS, et al. (2012) declara o aumento da responsabilização no processo de municipalização das ações do SUS quando destaca:

"A partir do gradual processo de implantação e descentralização intergovernamental do Sistema Único de Saúde (SUS), são requeridas novas competências e responsabilidades para a gestão governamental. Uma delas é a competência de governos em organizarem e utilizarem sistemas de Monitoramento e Avaliação (M\&A) de informações propícias para subsidiar processos decisórios, de planejamento, de prospecção logística, dentre outras possibilidades".

Sendo este confirmado por Preuss LT (2018) ao relatar que, apesar da expansão da capacidade gerencial entre as três esferas governamentais, a saúde é responsabilidade municipal. Todavia, Miranda AS, et al. (2012) e Reuter CLO, et al. (2016) citam vários aspectos do processo de monitoramento e avaliação, pautando que municípios pequenos tem maior dificuldade de realizar tais processos em saúde, ressalta ainda a falta de coesão entre os instrumentos de gestão e as informações geradas da base de dados das SMS, a falta de estrutura para desempenhar estas atividades sem equipe técnica para tal função e ainda a restrição desses processos a informações epidemiológicas e administração financeira enquanto que deveriam fazer parte de uma processo de análise e tomada de decisão.

Neste contexto, a descentralização trouxe obrigações à gestão municipal no sentido a estruturar-se para então cumprir desde o planejamento até os processos de avaliação. Para isto, um setor de primordial importância na implementação desses processos é a Vigilância em Saúde (VS) no sentido de que sua integração a Atenção Primária em Saúde (APS) vem contribuir para uma reorganização do processo do trabalho, principalmente na concepção do "território enquanto local privilegiado de atuação da atenção básica", de modo que a Vigilância em Saúde apresenta ferramentas que contribuem no planejamento, monitoramento e avaliação de ações e serviços de saúde (OLIVEIRA CM e CASANOVA AO, 2009; RECKTENWALDT M e JUNGES JR, 2017).

\section{O percurso da vigilância em saúde frente ao processo de integração com a Atenção Primária em Saúde.}

Netto GF, et al. (2017) traz em seu estudo o percurso da Vigilância em Saúde frente as acepções e expõe que, em 1968, foi adotado o conceito de vigilância populacional sendo a base para o conceito atual e define que a "vigilância é informação para ação". Discorre ainda sobre as primeiras medidas de Vigilância em Saúde no Brasil, no tempo colonial, e o Movimento da Reforma Sanitária Brasileira e a $8^{\text {a }}$ Conferência Nacional de Saúde onde define um conceito ampliado de saúde, e que responsabiliza o Estado quanto a Vigilância em Saúde. A criação do Sistema Único de Saúde, trouxe consigo novos arranjos institucionais no 
âmbito da Vigilância ao logo do tempo e, em 2003, se cria a Secretaria de Vigilância em Saúde (SVS), representada por cinco departamento, momento onde ganha força os processos de descentralização das ações da vigilância.

Frente a criação da SVS, a mesma passou a responsabilizar-se por todas as atuações administradas pelo SUS desde a vigilância epidemiológica de doenças à vigilância em saúde ambiental, surgindo com extremo apoio ao processo de descentralização e, intitulado, de acordo com a Portaria № 1.172/2004, complementar as ações realizada a nível estadual e municipal. Em 2006, a divulgação do Pacto Pela Saúde, baseado nas necessidades da população, estabelece a integração entre os setores e destaca seus objetivos, metas e benefícios (BRASIL, 2004; OLIVEIRA CM e CRUZ MM, 2015; TEIXEIRA MG, et al., 2018).

Nessa fase inicial, de 1999 a 2004, entende-se como implementação da vigilância à atenção primária e, a partir desse período, a segunda fase é marcada pelo fortalecimento da gestão e aprimoramento do planejamento e avaliação das equipes de saúde (OLIVEIRA CM e CRUZ MM, 2015; GUIMARÃES RM, et al., 2017). Desta forma, destaca o importante do papel da vigilância em saúde nas açõesdos serviços de saúde, sendo sua integração com a atenção primária um marco fundamental para organização do processo de trabalho das equipes no que diz respeito principalmente ao reconhecimento de seus problemas e definição de prioridades.

Embora exista diferentes normas e estratégias em todos os campos de gestão para integrar a Vigilância em Saúde à atenção básica, tal associação ainda não foi realizada de forma eficaz, sobretudo, no âmbito das equipes das Unidades Básicas de Saúde (UBS). Atualmente, a deficiente integração entre as áreas de conhecimento caracteriza umobstáculo frente a identificação de determinantes de saúde-doença e controle de agravos, contrapondo-se aos princípios propostos na Lei no 8.080 (BRASIL, 2018).

Considerando a necessidade de equipes capacitadas para avaliar e identificar, no contexto do território, possíveis riscos à saúde, o monitoramento e avaliação do processo de trabalho e, além disso, a indispensável definição das diretrizes e estratégias no contexto a gestão do SUS relacionado a vigilância, o Conselho Nacional de Saúde (CNS), por meio da Política Nacional de Vigilância em Saúde (PNVS), estabelece a transversalidade das ações da VS no processo saúde-doença contribuintes para integralidade na atenção à saúde o que, segundo a PNVS "pressupõe a inserção de ações de vigilância em saúde em todas as instâncias e pontos da Rede de Atenção à Saúde do SUS" (OKUMOTO O, et al., 2018; BRASIL, 2018). Neste sentido as equipes de saúde devem reconhecer as ações da VS como pertinentes a suas práticas no cuidado à saúde a fim de estabelecer um cuidado integral que corresponda as suas reais necessidades.

Oliveira CM e Casanova AO (2009) colocam que a "vigilância da saúde necessita estar apoiada em três pilares fundamentais: território, problemas e práticas de saúde", o que nos remete mais uma vez ao importante papel da integração APS e VS nas práticas de saúde. Também chamam atenção da territorialidade como ferramenta fundamental para organização do processo de trabalho da atenção básica (AB), que é o principal pilar da vigilância da saúde. Sendo ratificado também por Araújo GB, et al. (2017); um instrumento auxiliar no planejamento de ações para identificação de fatores sociais, ambientais e econômicos ligados aos problemas de saúde de uma área específica.

Existe uma enorme dificuldade em identificar os elementos que determinam o processo saúde-doença nos territórios, além de exercer o controle das doenças e agravos prioritários com a falta ou insuficiência na integração destas políticas. Conhecer os conceitos de território e territorialização se faz primordial diante ao cenário de prática e atuação da $A B$ e VS, bem como a organização de ações integradas de trabalho considerando a transversalidade, desde o olhar sobre o território até a organização das linhas de cuidado.

Existem ferramentas em usona $A B$ que são potentes no fortalecimento da integração prática, como a consulta individual e interconsulta, a discussão de casos e ações de matriciamento que poderá levar uma etapa seguinte onde se podem construir Projetos Terapêuticos Singulares (PTS) (BRASIL, 2018). Ainda como instrumento de atenção primária, o genograma e o ecomapa também auxiliamo fortalecimento da integralidade e, além disso, as atividades de grupo, a busca ativa e a notificação de doenças e agravos, a 
visita e o atendimento domiciliar, bem como intervenções comunitárias como ações de imunização, além de ferramentas de apoio matricial e suporte à distância como o teles saúde (ARAÚJO GB, et al., 2017; BRASIL, 2018). Nessa perspectiva, a inserção da VS na $A B$ traz contribuições significativas a todos os membros $e$ equipes dos serviços de saúde, no entanto, faz-se necessário planejamento avaliação dos recursos.

O Ministério da Saúde, através Política Nacional de Atenção Básica (2017), dispõe da necessidade do processo de institucionalizar o monitoramento e avaliação e dos serviços de saúde com propósito de qualificar tal ação (BRASIL, 2017). Para Nicola T e Pelegrini AHW (2018) a "institucionalização da avaliação representa uma estratégia importante a ser promovida para melhorar a eficiência no sistema de saúde". Desse modo, traz o importante papel da gestão de trabalho, baseando-se em conhecimentos sólidos e criando uma cultura de avaliação, com vistas a resultados que tragam melhoria ao sistema de saúde e, por conseguinte, a satisfação do usuário como resultado de melhores condições de saúde.

De acordo com Carvalho ALB, et al. (2012) e Nicola T e Pelegrini AHW (2018) a institucionalização forma novas responsabilidades e competências a nível governamental, possibilitando negociações entre gestores com vista no meio tecnológico e instrumental com objetivo de melhorar processo de decisão, sendo este o propósito primordial da gestão em saúde, adequando-o as especificidades dos sistemas de saúde.

Carvalho ABL e Shimizu HE (2017) traz a institucionalização da avaliação como um movimento pendular de descentralização/centralização que tem sido destaque quanto a elaboração e implementação e políticas sob as obrigações da gestão em cada esfera de governo. A finalidade das avaliações vai além das condições de intervenções, sendo com ou sem êxito, remete a este processo o aprendizado contínuo visando o amadurecimento da gestão. No entanto, expõe tal avaliação como paradoxo ao relatar desafios por parte dos gestores em chegar ao consenso quanto aos resultados das avaliações, uma vez que possuem ocupações distintas e, com isso, é necessário, de início, aprimorar os mecanismos de pactuação (DENIS JL, 2010; CARVALHO ABL e SHIMIZU HE, 2017). Cabe ressaltar, o acesso aos resultados referente as avaliações e monitoramento se estabelece como uma importante base para democratização da informação de gestão da $A B$, caracterizando assim a transparência dos serviços.

\section{CONSIDERAÇÕS FINAIS}

Frente aos desafios da ampliação no plano de Atenção Primária em Saúde, quanto a proposta de integração da Vigilância em Saúde à Saúde básica e a gestão desses recursos, destaca a necessidade de inovação dos métodos e modelos de planejar e avaliar para auxiliar no processo decisório de determinada problemática, além de subsidiar na reorganização do processo do trabalho, ações e serviços de saúde. Diante desta perspectiva, esse estudo expõe a complexidade da atenção básica e pretende auxiliar profissionais, pesquisadores e gestores a entender o processo de integralidade entre áreas e seus benefícios, além de contribuir para debates acerca competências e estratégias entre os setores da gestão em saúde e, de certo modo, nortear condutas no sentido ao aprimoramento das avaliações dos serviços de saúde, com visão no melhor funcionamento do Sistema.

\section{REFERÊNCIAS}

1- ARAÚJO GB, et al. Territorialização em saúde com instrumento de formação para estudantes de medicina: relato de experiência. SANARE, Sobral, 2017; 16(1): 124-129.

2- BRASIL. Lei № 8.142 , de 28 de dezembro de 1990. Dispõe sobre a participação da comunidade na gestão do Sistema Único de Saúde (SUS\} e sobre as transferências intergovernamentais de recursos financeiros na área da saúde e dá outras providências. Brasilia,1990.

3- BRASIL. Lei no 8.080, de 19 de setembro de 1990. Lei Orgânica da Saúde. Dispõe sobre as condições para a promoção, proteção e recuperação da saúde, a organização e o funcionamento dos serviços correspondentes e dá outras providências. Brasília, 1990.

4- BRASIL. Ministério da Saúde. Portaria № 1.172, de 15 de junho de 2004 Regulamenta a NOB SUS 01/96 no que se refere às competências da União, Estados, Municípios e Distrito Federal, na área de Vigilância em Saúde, define a sistemática de financiamento e dá outras providências, 2004.

5- BRASIL. Ministério da Saúde. Cadernos HumanizaSUS: atenção básica. Brasília, DF, 2010.

6- BRASIL. Ministério da Saúde. Política Nacional de Atenção Básica Operacionalização, 2010. 
7- BRASIL. Ministério da Saúde. Portaria GM № 1.378, de 9 de julho de 2013. Regulamenta as responsabilidades e define diretrizes para execução e financiamento das ações de Vigilância em Saúde pela União, Estados, Distrito Federal e Municípios, relativos ao Sistema Nacional de Vigilância em Saúde e Sistema Nacional de Vigilância Sanitária. Diário Oficial da União, 2013.

8- BRASIL. Ministério da Saúde. PORTARIA № 2.436, de 21 de setembro de 2017. Aprova a Política Nacional de Atenção Básica, estabelecendo a revisão de diretrizes para a organização da Atenção Básica, no âmbito do Sistema Único de Saúde (SUS). Diário Oficial da União, 2017.

9- BRASIL. Ministério da Saúde. Sistema Único de Saúde (SUS): estrutura, princípios e como funciona, 2017.

10- BRASIL. Ministério da Saúde. Secretaria de Atenção à Saúde. Secretaria de Vigilância em Saúde. Guia Política Nacional de Atenção Básica - Módulo 1: Integração Atenção Básica e Vigilância em Saúde, 2018.

11- BRASIL. Ministério da Saúde. Conselho Nacional de Saúde. Resolução MS/CNS no 588, de 12 de julho de 2018. Fica instituída a Política Nacional de Vigilância em Saúde (PNVS), aprovada por meio desta resolução. Diário Oficial da República Federativa do Brasil, Brasília (DF), 2018.

12- BRASIL. Ministério da Saúde. Conselho Nacional de Saúde. Resolução MS/CNS n 588, de 12 de julho de 2018. Fica instituída a Política Nacional de Vigilância em Saúde (PNVS), aprovada por meio desta resolução. Diário Oficial da República Federativa do Brasil, Brasília (DF); ago 13, 2018.

13- BOUSQUAT AEM, et al. Tipologia da estrutura das unidades básicas de saúde brasileiras: os 5 R. Cad. Saúde Pública, 2017; 33(8).

14- CARVALHO ALB, SHIMIZU HE. A institucionalização das práticas de monitoramento e avaliação: desafios e perspectivas na visão dos gestores estaduais do Sistema Único de Saúde (SUS). Interface (Botucatu), 2017; 21(60): 23-33.

15- CARVALHO ALB, et al. A gestão do SUS e as práticas de monitoramento e avaliação: possibilidades e desafios para a construção de uma agenda estratégica. Ciência \& Saúde Coletiva, 2012; 17(4): 901-911.

16- DENIS JL. Institucionalização da avaliação na administração pública. Rev Bras Saude Mater Infant, 2010.

17- FACCHINI LA, et al. Qualidade da Atenção Primária à Saúde no Brasil: avanços, desafios e perspectivas. Saúde Debate. Rio de Janeiro, 2018; 42: 208-223.

18- FACCHINI LA. A Declaração de Alma-Ata se revestiu de uma grande relevância em vários contextos. Escola Politécnica de Saúde Joaquim Venâncio, 2018.

19- FURTADO JP, et al. Planejamento e Avaliação em Saúde: entre tagonismo e colaboração Cad. Saúde Pública, 2018; 34(7).

20- GIOVANELLA L. Atenção básica ou atenção primária à saúde? Cad. Saúde Pública, 2018.

21- GUIMARÃES RM, et al. Os desafios para a formulação, implantação e implementação da Política Nacional de Vigilância em Saúde. Ciência \& Saúde Coletiva, 2017; 22(5): 1407-1416.

22- INSTITUTO DE PESQUISA ECONÔMICA APLICADA (IPEA). Federalismo, Integralidade E Autonomia no SUS: Desvinculação Da Aplicação De Recursos Federais E Os Desafios Da Coordenação. Brasília. Rio de Janeiro, 2018.

23- LORENZETTI J, et al. Gestão em saúde no Brasil: diálogo com gestores públicos e privados. Texto contexto enferm. Florianópolis, 2014; 23(2).

24- MIRANDA AS, et al. Subsídios sobre práticas de monitoramento e avaliação sobre gestão governamental em Secretarias Municipais de Saúde. Ciência \& Saúde Coletiva, 2012; 17(4): 913-920.

25- NICOLA T, PELEGRINI AHW. Avaliação em saúde nos serviços de atenção primária no Brasil: revisão integrativa da literatura. J Nurs Health, 2018; 8(1).

26- NETTO GF, et al. Vigilância em Saúde brasileira: reflexões e contribuição ao debate da 1ª Conferência Nacional de Vigilância em Saúde. Ciência \& Saúde Coletiva, 2017; 22(10): 3137-3148.

27- OLIVEIRA CM, CASANOVA AO. Vigilância da saúde no espaço de práticas da atenção básica. Ciência \& Saúde Coletiva, 2009; 14(3): 929-936.

28- OLIVEIRA CM, CRUZ MM. Sistema de Vigilância em Saúde no Brasil: avanços e desafios. Rio de Janeiro, 2015; 39(104): 255-267.

29- OKUMOTO O, et al. A Política Nacional de Vigilância em Saúde. Epidemiol. Serv. Saúde, 2018; 27(3).

30- PINAFO E, et al. Descentralização da gestão: caminho percorrido, nós críticos e perspectivas. Ciênc. Saúde colet, 2016; 21 (5).

31- PINHEIRO ALS, et al. Gestão da saúde: o uso dos sistemas de informação e o compartilhamento de conhecimento para a tomada de decisão. Texto contexto - enferm. Florianópolis, 2016; 25(3).

32- POLATI AM, et al. Os desafios da gestão do SUS sob a ótica de gestores municipais de saúde. JMPHC. Journal of Management and Primary Health Care, 2016; 7(1): 2-2.

33- PREUSS LT. A gestão do Sistema Único de Saúde no Brasil e as regiões de fronteira em pauta.R. Katál., Florianópolis, 2018; 21(2): 324-335.

34- ROUTER CLO, et al. Práticas de monitoramento na gestão municipal em saúde e sua interface com a enfermagem. Rev Gaúcha Enferm, 2016; 37.

35- RECKTENWALDT M, JUNGES JR. A organização e a prática da Vigilância em Saúde em municípios de pequeno porte. Saúde Soc. São Paulo, 2017: 26(2): 367-381.

36- SOUZA MMO, GUEDES JPF. A integração da vigilância em saúde com a atenção básica por meio da estratégia saúde da família, 2013.

37- TEIXEIRA MG, et al. Vigilância em Saúde no SUS - construção, efeitos e perspectivas. Ciência \& Saúde Coletiva, 2018: 23(6): 1811-1818.

38- VALADÃO APS, et al. Melhor no passado: a verdadeira saúde da família. Saúde soc. São Paulo, 2019: 28(1). 